\title{
From single-trial EEG to brain area dynamics
}

\author{
A. Delorme ${ }^{\mathrm{a}, *}$, S. Makeig ${ }^{\mathrm{a}}$, M. Fabre-Thorpe ${ }^{\mathrm{b}}$, T. Sejnowski ${ }^{\mathrm{a}}$ \\ ${ }^{a}$ The Salk Institute for Biological Studies, 10010 N. Torey Pines Read, La Jolla, CA 92109, USA \\ ${ }^{\mathrm{b}}$ Centre de Recherche Cerveau and Cognition (CERCO) (UMR 5549), Faculte de medecine, \\ 133 route de Narbonne, 31062 Toulouse Cedex, France
}

\begin{abstract}
We here present a new technique for visualizing the temporal dynamics of brain area activation and interaction at high-temporal resolution. We first applied independent component analysis to concatenated single-trial EEG data from a fast go-nogo categorization task of natural images and showed that individual independent components might index neural synchrony within and between intracranial brain sources. We used time-frequency decomposition to model their dynamic interactions. In particular, following stimulus presentations, we showed that several independent components were activated and synchronized in the theta frequency range (near $4 \mathrm{~Hz}$ ). (c) 2002 Elsevier Science B.V. All rights reserved.
\end{abstract}

Keywords: EEG \& ERP; Visual categorization; ICA; Time-frequency analysis; Brain areas' synchronizations

\section{Introduction}

None of the current functional imaging techniques is well suited to address the dynamics of brain source activity and their dynamic relationships during visual perception and categorization. Only EEG has the sufficient time resolution to capture the macroscopic dynamics of brain activation and synchronization. However, using EEG we face problem of reconstructing the intracranial brain sources from the observed signals: the projections on the scalp of separately generated EEG processes typically overlap both in time and space, becoming inextricably mixed in recordings from scalp electrodes. Recovering the locations and activities of these brain processes is known as the EEG

\footnotetext{
* Corresponding author. Centre de Recherche Cerveau and Cognition (CERCO) (UMR 5549), Faculte de medecine, 133 route de Narbonne, 31062 Toulouse Cedex, France. Tel.: +33-562-173-774; fax: +33-562-172-809.

E-mail addresses: arno@salk.edu (A. Delorme),scott@salk.edu (S. Makeig),mft@cerco.ups-tlse.fr (M. Fabre-Thorpe), terry@salk.edu (T. Sejnowski).
} 
inverse problem. To avoid fully solving this problem, one can compute differences between stimulus-locked averages of sets of trials differing by only one characteristic. The remaining difference wave average may then be more easily interpreted [12]. However, this technique assumes that the difference is composed of activity from a few easily separable brain electrical sources, which may not be the case.

Here we investigated the potential of a new statistical technique, independent component analysis (ICA), to separate EEG sources mixed at scalp electrodes and to help solve the inverse problem [1,6]. We showed that individual independent components may index neural synchrony in intracranial brain sources. We illustrate their dynamics and time-varying coherence following stimulus presentation.

\section{Material and methods}

We used EEG data recorded during a rapid go/nogo visual categorization task using natural photographs [2]. Subjects were presented with photographs, half of which included an animal for $20 \mathrm{~ms}$ at random intervals of $1.8-2.2 \mathrm{~s}$. Subjects were asked to release a held button whenever a presented photograph contained an animal image. Overall performance was high both in terms of accuracy (94\% correct responses) and speed (median reaction time $440 \mathrm{~ms}$ ). Moreover, though the task involved complex visual processing, averaged EEG epochs for target and non-target stimuli differed significantly as early as $150 \mathrm{~ms}$ after stimulus onset [11]. Thus, despite the complexity of the task, it appeared to involve automated processing in the visual system. For this reason we believe the task is well suited for studying the dynamics of brain electric fields during visual processing.

To decompose the data into brain source activities, we used an infomax ICA algorithm. ICA algorithms find a coordinate frame onto which the projection of the data has minimal temporal overlap. The core mathematical concept of ICA is to minimize mutual information among the data projections. ICA can be viewed as a linear decomposition alternative to principal component analysis (PCA). In PCA, the data is represented using perpendicular axes, whereas ICA is not limited by this constraint [7]. ICA has been applied to various problems including decomposing fMRI data and performing speech and noise separation [9]. However, performing ICA decompositions is most appropriate when sources are linearly mixed in the recorded signal, which is precisely the case for the various brain processes summed by volume conduction in scalp EEG.

Some earlier studies applied ICA to collections of single-trial EEG data averages, but this raises several problems. First, ICA may require many observations to separate two or more processes, so a problem often faced using averaged EEG data is that there are not enough conditions in the training set to obtain stable ICA components. Another problem with using averaged EEG data is that the averaging process may cancel out the activity of many brain sources. Finally, data averages by their nature contain sums of activity occurring at similar times. When two or more sources reliably contribute to the response average at the same times, ICA may assign their sum to a single component. 
(a) topography
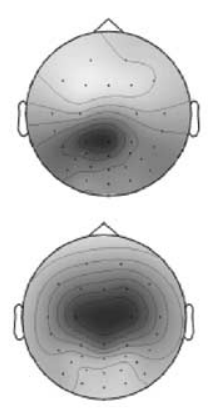

(b) activity

Trials
250

Component 3

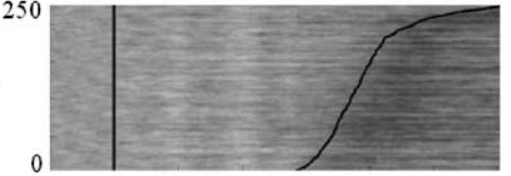

Component 2

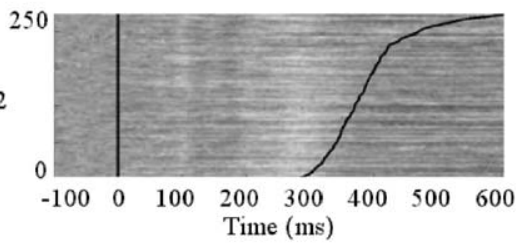

(c) localization
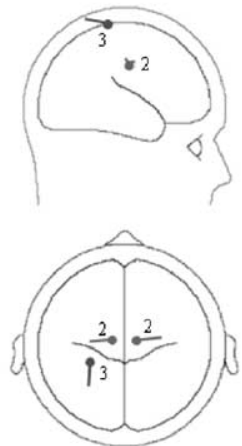

(d) Time-frequency power (top) and Inter-trial coherence (bottom)
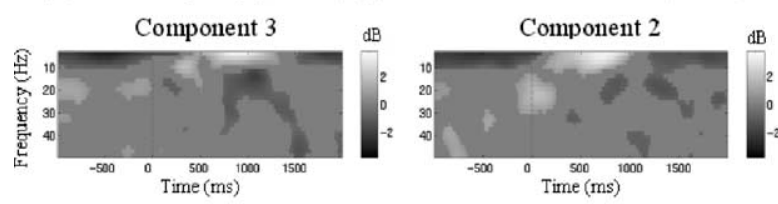

(e) Cross-coherence amplitude (top) and phase (bottom)
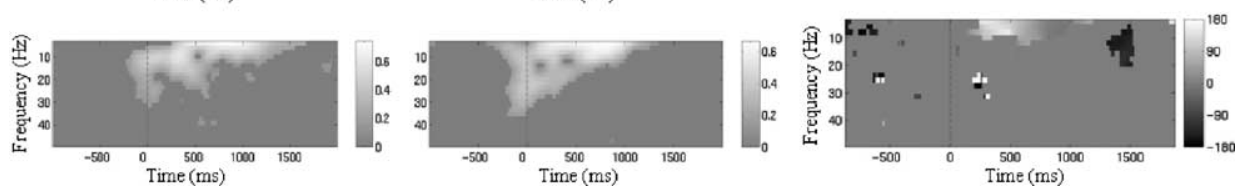

Fig. 1. Properties of two independent components (2 and 3). (a) Component scalp map (shading indicates relative projection strength, arbitrary units). (b) ERP-image plots of activity of stimulus-locked trials' target response of component 2 and 3 (shading indicates activity, arbitrary units). The trials are ordered (from top to bottom) according to the reaction time of the subject (black thick line). Plots (a) and (b) show the spatial and temporal patterns constitutive of the two components. (c) Equivalent dipole location for components 2 and 3 found by BESA. See Fig. 2 concerning the accuracy of these models. (d) Spectral power and inter-trial coherence changes following target stimuli. The top panels indicate the power spectral perturbations (in $\mathrm{dB}$, relative to pre-stimulus baseline); the bottom panels show the inter-trial coherence (from 0 to 1 ) (see text for details). (e) Cross-coherence between components 2 and 3. Top panel: cross-coherence magnitude (from 0 to 1 ) indicating the amount of synchronization of the two components at each frequency and time window. Bottom panel: cross-coherence phase indicating which of the 2 components is leading (phase in degrees). These time/frequency measures may be used to visualize the global interaction between selected independent components in a specific frequency band (see Fig. 3).

To tackle these problems, we chose to train ICA on the whole collection of correct single-trial data from each subject [5]. More specifically, we used the infomax ICA algorithm of the ICA/EEG toolbox of Makeig et al. [6]. After applying ICA, we manually $[3,4]$ or semi-automatically distinguished those components that were related to neural activity from those related to artifacts. Software we developed for artifact identification is available at www.sccn.ucsd.edu/eeglab. Each independent component consists of a time course of its activity (Fig. 1b) and a scalp topography (Fig. 1a) specifying its projection to the electrodes. From the non-artifact independent components, we choose 
the six for each subject that contributed the most to the early averaged visual response (from 0 to $400 \mathrm{~ms}$ ).

\section{Results}

Most neural activity-related independent component could be well modeled using a simple intracranial electrical source configuration (Fig. 1c) by a single brain equivalent dipole or by two bilateral and symmetrical equivalent dipoles in a spherical head model using the BESA software [8]. It is important to note that the ICA algorithm only relies on statistics and knows nothing about the biophysics or geometry of the head. The positions of the electrodes are irrelevant for the algorithm, and shuffling the time order of the data also does not alter the resulting decomposition. As a consequence, the fact that most independent components were accounted by single equivalent dipoles with low residual variance suggests that these components likely represent the activity of unitary (or in some cases possibly bilateral) brain sources. Among the six selected independent components for the subject shown, three $(4,5,6)$ had equivalent dipole locations in or near visual cortex, two $(1,2)$ had pre-central midline equivalent dipole locations, and one (3) had an equivalent dipole location near the right hand motor cortex. When localizations were calculated separately for two task sessions for this subject, there was a clear correspondence between the equivalent dipole locations for the two sessions (Fig. 2).

We then applied time-frequency analysis to the activity time course of the six selected independent components to detect the occurrence of spectral power changes at specific frequencies and times relative to stimulus onset. We used single-cycle wavelets to obtain a better time resolution than standard Fourier transforms (we verified that the results using the two methods were similar). Fig. 1 ( $d$ and e upper panels) shows the dynamics of two of the selected components. All changes shown were significant at the $p<0.01$ level according to a bootstrap test using surrogate data. We observed that activity of the six components varied with time and conditions in several frequency bands. Several of the selected independent components became more active in the low theta frequency band (near $4 \mathrm{~Hz}$ ) following both target and non-target stimuli (Fig. 3). At this frequency, it appeared that visual brain components were first to increase their activity in this frequency band, then midline components and finally the motor area component. The motor component became more active only in target trials, consistent with the fact that the subject was instructed to give a motor response only following target images. We also found a similar pattern of independent component activations at the same frequency in data from the same subject performing the task on a different day (data not shown). The method allows close study of component activities in any frequency range.

To determine the consistency of these changes across trials in the selected independent components, we used inter-trial coherence, a measure of the reproducibility of the phase of stimulus-locked trial activity at each frequency (for details about these measures see [7]). Fig. 1 (d and e lower panels) illustrates inter-trial coherence results for two of the selected independent components. Fig. 3 represents the time course of 


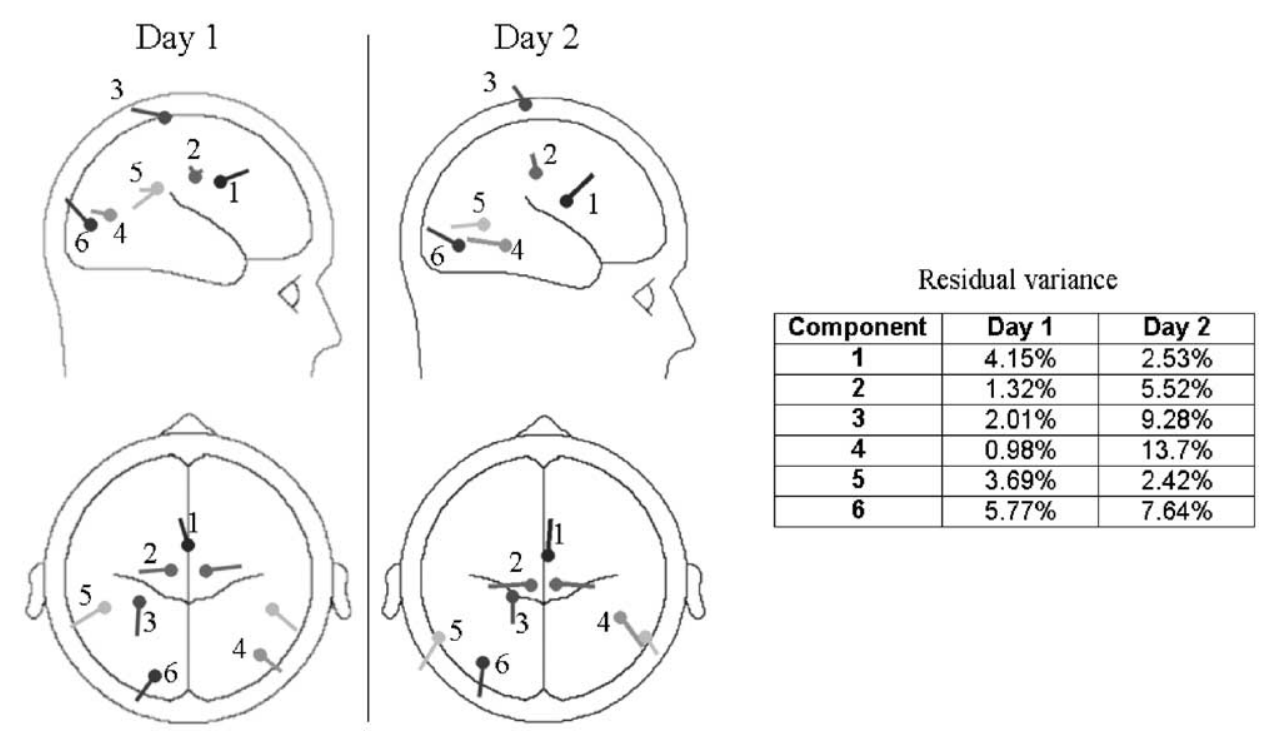

Fig. 2. Equivalent dipole location of the six selected independent components contributing to the evoked response of the same subject in two different sessions. Across sessions correspondences are indicated. On the right, the table indicates the accuracy of the model for all selected components (residual variance unaccounted for in the BESA spherical head model). For each component we chose the better fit of two models: a single equivalent dipole model and two bilateral equivalent dipoles constrained to left/right symmetry. All electrodes were considered in the localization of the equivalent dipoles (except at most 2 pairs of bilateral noisy temporal electrodes in some cases). Among the equivalent dipoles, 3 could be interpreted as being in or near the visual cortex $(4,5,6)$, two along the pre-central midline $(1,2)$ and one near the right hand motor cortex (3).

inter-trial coherence (and power and cross-coherence) in low theta frequency range for the six selected components. With respect to this measure, each component had a specific behavior. For instance, stimulus-locked inter-trial coherence for component 6 at $300 \mathrm{~ms}$ was higher following non-target stimuli than following target stimuli.

Though the independent components returned by ICA were maximally independent, we observed that they were not totally independent. The amplitude of the cross-coherence value at a given frequency between stimulus-locked trial activity of two components indexed the amount of synchronization of these components at that frequency. The phase of the cross-coherence indicated which of the two components' activities tended to lead at that frequency. For instance, a high cross-coherence amplitude and zero phase difference for two components at $4 \mathrm{~Hz}$ would indicate that they tended to be highly synchronized in that frequency range. Fig. 3 visualizes significant synchronizations between all pairs of the six selected independent components for target and non-target trials near $3.6 \mathrm{~Hz}$ using broken lines connecting them. Overall, the synchronization of the selected components was higher following stimulus presentation (100-500 ms) than during the pre-stimulus baseline or thereafter (before $0 \mathrm{~ms}$ or after $500 \mathrm{~ms}$, data not shown). We also observed both event-related synchronization and desynchroniza- 


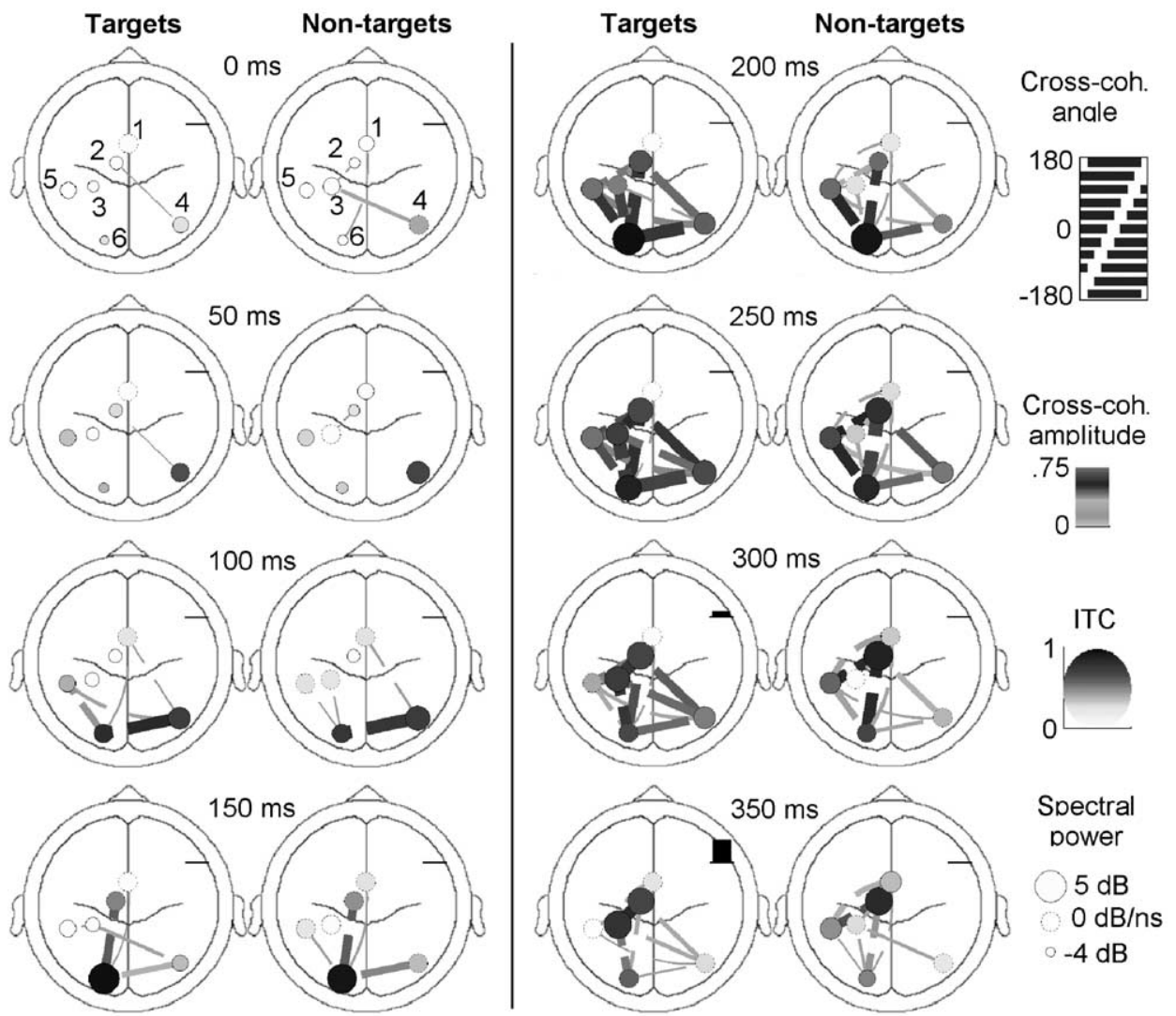

Fig. 3. Frames from an animation showing the activation of the independent components and their cooperativity in the low theta range frequency during visual processing (from 0 to $350 \mathrm{~ms}$ ) of target and non-target stimuli in the low theta frequency band $(3.6 \mathrm{~Hz})$. For details on the indicated components, see Fig. 2. Relative spectral power (in $\mathrm{dB}$, relative to pre-stimulus baseline) is visualized by the size of the circles, whereas the shading of the circles represents the strength of inter-trial coherence (ITC). The thickness and color of the lines connecting each pair of components illustrate the magnitude of their synchronization (cross-coherence amplitude from 0 to 1 ). The gap in the lines connecting two components shows the relative phase of the two components' activity (cross-coherence phase in degree). If this gap is centered, it indicates that the two components' activity have zero phase difference and tend to be synchronous in this frequency range. The histogram, on the upper part of each head plot, indicates the relative probability of response of the subject. In this example one can clearly see that peaks in power occurs at different times with respect to stimulus onset for the different selected components, and the appearance of theta synchronization among the components (peaking near $250 \mathrm{~ms}$ ). A movie of this figure is available at www.cnl.salk.edu/ arno/movies.html together with Matlab programs used to generate the animations.

tion between independent components. As an example, the phase relationship of two components with occipital equivalent dipole locations (components 4 and 6) changed across time: from a tendency towards synchronization near $150 \mathrm{~ms}$, the two components tended to become anti-synchronized by $200 \mathrm{~ms}$ (Fig. 3). 


\section{Discussion}

We have shown that the use of an advanced set of signal-processing tools can reveal the dynamics of brain activation and synchronization phenomena at high-temporal resolution which otherwise appeared to be inextricably embedded in the multi-channel EEG signals and are not revealed by standard averaging methods. In the visual categorization task, we showed for one subject across two sessions, a reproducible pattern of activation at several brain locations in a low-frequency range. Changes in EEG activity appeared in and between cortical visual areas, pre-central midline areas and a motor-related cortical area. We believe further study of the result of our analyses can cast new light on the debate about feedforward and feedback hypotheses of visual processing [10-12].

The mathematical tools we used are still unconventional in neuroscience. However, infomax ICA is now widely used by the scientific community, and the spectral measures of coherence and cross-coherence we used are standard techniques for determining the relationship of two or more electrical sources. The method presented here allows us to tackle the complex dynamics and relationships of brain intracranial equivalent dipole locations of independent components. While it might be still too early for us to interpret the observed coherences and synchronizations, our results indicate the potential utility of these techniques over traditional approaches. In conventional EEG analyses, spectral analyses are applied at the electrode level, including measures of synchrony between scalp electrodes using cross-coherence [13]. However, the interpretation of electrode coherences are ambiguous as they may be produced by various amplitude changes in several processes at different brain locations. For example, coherence increases between two electrodes may be accounted for by an increase in the power of a single major EEG sources projecting to both electrodes, and thus synchronizing the recorded activity. In our case, computing cross-coherence between components means we may more directly assess the dynamic relationships between different brain areas.

\section{Acknowledgements}

This research was supported by the "Institut National de Recherche en Informatique et Automatique" of France, by the Howard Hughes Medical Institute, the National Institutes of Health, USA and by the Swartz foundation.

\section{References}

[1] A.J. Bell, T.J. Sejnowski, An information-maximization approach to blind separation and blind deconvolution, Neural Comput. 7 (1995) 1129-1159.

[2] M. Fabre-Thorpe, A. Delorme, C. Marlot, S. Thorpe, A limit to the speed of processing in ultra-rapid visual categorization of novel natural scenes, J. Cogn. Neurosci. 13 (2001) 171-180.

[3] T.P. Jung, S. Makeig, C. Humphries, T.W. Lee, M.J. McKeown, V. Iragui, T.J. Sejnowski, Removing electroencephalographic artifacts by blind source separation, Psychophysiology 37 (2000) 163-178. 
[4] T.P. Jung, S. Makeig, M. Westerfield, J. Townsend, E. Courchesne, T.J. Sejnowski, Removal of eye activity artifacts from visual event-related potentials in normal and clinical subjects, Clin. Neurophysiol. 111 (2000) 1745-1758.

[5] T.P. Jung, S. Makeig, M. Westerfield, J. Townsend, E. Courchesne, T.J. Sejnowski, Analysis and visualization of single-trial event-related potentials, Hum. Brain Mapp. 14 (2001) 166-185.

[6] S. Makeig, A.J. Bell, T.P. Jung, T.J. Sejnowski, Independent component analysis of electroencephalographic data, Neural Inform. Proc. System (1996) 145-151.

[7] S. Makeig, M. Westerfield, T.P. Jung, J. Covington, J. Townsend, T.J. Sejnowski, E. Courchesne, Functionally independent components of the late positive event-related potential during visual spatial attention, J. Neurosci. 19 (1999) 2665-2680.

[8] W. Miltner, C. Braun, R. Johnson Jr., G.V. Simpson, D.S. Ruchkin, A test of brain electrical source analysis (BESA): a simulation study Electroencephalogr. Clin. Neurophysiol. 91 (1994) 295-310.

[9] H.-M. Park, H.-Y. Jung, T.-W. Lee, S.-Y. Lee, On subband-based blind signal separation for noisy speech recognition, Electron. Lett. 35 (1999) 2011-2012.

[10] E. Rodriguez, N. George, J.P. Lachaux, J. Martinerie, B. Renault, J. Varela, Perception's shadow: long-distance synchronization of human brain activity, Nature 397 (1999) 430-433.

[11] S.J. Thorpe, M. Fabre-Thorpe, Seeking categories in the brain, Science 291 (2001) 260-263.

[12] S. Thorpe, D. Fize, C. Marlot, Speed of processing in the human visual system, Nature 381 (1996) 520-522.

[13] F. Varela, J.P. Lachaux, E. Rodriguez, J. Martinerie, The brainweb: phase synchronization and large-scale integration, Nat. Rev. Neurosci. 2 (2001) 229-239. 